International Journal of Pure and Applied Mathematics

Volume 107 No. 1 2016, 69-86

ISSN: 1311-8080 (printed version); ISSN: 1314-3395 (on-line version)

url: http://www.ijpam.eu

doi: 10.12732/ijpam.v107i1.7

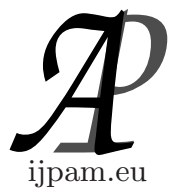

\title{
NEW EFFICIENT PHASE-FITTED AND AMPLIFICATION-FITTED RUNGE-KUTTA METHOD FOR OSCILLATORY PROBLEMS
}

\author{
Firas Adel $^{1,2} \S$, Norazak Senu ${ }^{1,3}$ Fudziah Ismail ${ }^{1,3}$, \\ Zanariah Abd. Majid ${ }^{1,3}$ \\ ${ }^{1}$ Department of Mathematics \\ Faculty of Science \\ Universiti Putra Malaysia \\ 43400 UPM, Serdang, Selangor, MALAYSIA \\ ${ }^{2}$ Department of Mathematics \\ Faculty of Computer Science and Mathematics \\ Tikrit University, Sallah AL-Deen, IRAQ \\ ${ }^{3}$ Institute for Mathematical Research \\ Universiti Putra Malaysia \\ 43400 UPM, Serdang, Selangor, MALAYSIA
}

\begin{abstract}
A new Runge-Kutta (RK) method is constructed to solve first-order differential equations with oscillatory solutions. This new method is based on the Runge-Kutta method of order four with seven-stage. Numerical tests are performed, and the results of the new method is compared with the existing methods. The numerical results show that the new method is more efficient.
\end{abstract}

AMS Subject Classification: 65L05, 65L06

Key Words: Runge-Kutta methods, phase-fitted, amplification-fitted and oscillatory problems

Received: January 4, 2016

Published: March 23, 2016

$\S$ Correspondence author
(C) 2016 Academic Publications, Ltd. url: www.acadpubl.eu 


\section{Introduction}

In this study, we focus on the initial value problems (IVPs) of the form:

$$
y^{\prime}(x)=f(x, y), y\left(x_{0}\right)=y_{0}
$$

where the solutions of the ordinary differential equations (1) are oscillating. This type of problem can be found in many fields of applied science such as physical chemistry, astronomy, quantum mechanics, mechanics, and electronics. Some authors have suggested adapting traditional integration for the oscillatory character of the solution to problem (1). Bettis [1] constructs three- and four-stage methods that solve the equation $y^{\prime}=i w y$ without truncation error. Franco [2] improves the update of Runge-Kutta Nyström methods adapted to the numerical integration of perturbed oscillators. Anastassi and Simos [3] construct phase- and amplification-fitted explicit RK methods for the numerical solution of orbital problems. Many researchers focus on the effective numerical integration of specific categories of oscillatory problems, such as Vigo-Aguiar and Simos [4] constructed exponentially and trigonometrically-fitted methods for orbital problems.

This current work focus on phase-fitted and amplification-fitted RK integrators whose coefficients depend on the product of fitting frequency $w$ and step size $h$. Section 2 introduces the notion of phase-fitted and amplification-fitted RK-type methods and derivation of the conditions. In Section 3, new method of order four is constructed. We compare the results with existing methods in Section 4.

\section{Phase-Fitted and Amplification-Fitted Conditions}

An $s$-stage Runge-Kutta method:

$$
\begin{gathered}
y_{n+1}=y_{n}+h \sum_{i=1}^{s} b_{i}(z) k_{i} \\
k_{i}=f\left(x_{n}+c_{i} h, y_{n}+h \sum_{j=1}^{i-1} a_{i j} k_{j}\right), i=1, \ldots, s .
\end{gathered}
$$

where $c_{i}, a_{i j}, i, j=1, \ldots, s$ are real numbers, $h$ is step size, and $b_{i}(z), i=1, \ldots, s$ are even functions of $z=w h$. Scheme 2 can be represented by the Butcher tableau below: 


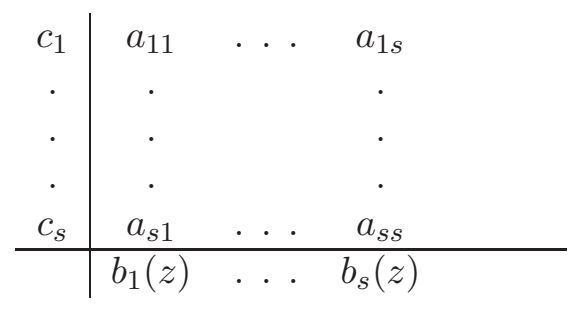

Hairer et al [5], explained the order conditions for the RK method (2) which can be derived by just considering the autonomous equation $y^{\prime}=f(y)$.

Van der Houwen and Sommeijer [6] and Paternoster [7] suggested apart from the algebraic order, the analysis of phase-lag and dissipation is important. We consider the following linear scalar equation:

$$
y^{\prime}=i w y \text {. }
$$

The exact solution of this equation with the initial value $y\left(x_{0}\right)=y_{0}$ satisfies

$$
y\left(x_{0}+h\right)=R(z) y_{0}
$$

where $R(z)=\exp (z)$. This means that after a period of time $h$, the exact solution experiences a phase advance $z=h w$ and the amplification remains constant.

When applying the RK method (2) to (3) yield

$$
\begin{gathered}
y_{n+1}=R(z) y_{0}, \\
R(z)=1+z b^{T}(I-z A)^{-1} e .
\end{gathered}
$$

where

$$
e=(1, \ldots, 1)^{T}, A=\left[a_{i j}\right] \text { and } b^{T}=\left[b_{1}, b_{2}, b_{3}, \ldots, b_{s}\right] .
$$

The numerical solution attain a phase advance $\arg R(z)$ and the amplification factor $|R(z)| . R(z)$ is called the stability function of the method (2). Denote the real and imaginary part of $R(z)$ by $U(z)$ and $V(z)$ respectively. Then, for small $h$ we have

$$
\begin{gathered}
U(z)=1-z^{2}\left(b^{T} A e\right)+z^{4}\left(b^{T} A^{3} e\right)-z^{6}\left(b^{T} A^{5} e\right)+\ldots \\
V(z)=z\left(b^{T} e\right)-z^{3}\left(b^{T} A^{2} e\right)+z^{5}\left(b^{T} A^{4} e\right)-z^{7}\left(b^{T} A^{6} e\right)-\ldots .
\end{gathered}
$$

For small $h, \arg R(z)=\tan ^{-1}\left(\frac{V(z)}{U(z)}\right)$ and $|R(z)|=\sqrt{U^{2}(z)+V^{2}(z)}$. 
Van der Houwen and Sommeijer [6] stated, the quantities

$$
P(z)=v-\arg R(z), D(v)=1-|R(z)|
$$

which are called the phase-lag (or dispersion) and the error of amplification factor (or dissipation) of the method, respectively. If

$$
P(z)=O\left(z^{q+1}\right), D(z)=O\left(z^{p+1}\right),
$$

then the method is called dispersive of order $q$ and dissipative of order $p$, respectively. If

$$
P(z)=0, D(z)=0,
$$

the method is called phase-fitted (or zero-dispersive) and amplificationfitted (or zero-dissipative), respectively. It is interesting to consider the phase properties of the update of the scheme (2). Suppose that the internal stages have been exact for the linear equation (3), that is, $Y_{i}=\exp \left(c_{i} z\right) y_{0}$, then the update gives

$$
R(z)=1+z \sum_{i=1}^{s} b_{i}(z) \exp \left(c_{i} z\right) .
$$

Denote the real and imaginary part of $R(z)$ by $U(z)$ and $V(z)$, respectively. Then, for small $h$.

$$
\begin{gathered}
U(z)=1-z \sum_{i=1}^{s} b_{i}(z) \sin \left(c_{i} z\right) \\
V(z)=z \sum_{i=1}^{s} b_{i}(z) \cos \left(c_{i} z\right)
\end{gathered}
$$

Theorem 1. The method (2) is phase-fitted and amplification-fitted if and only if

$$
U(z)=\cos (z), \quad V(z)=\sin (z)
$$

\section{Construction of New Method}

In this section, we consider a seven-stage RK method as given in Butcher [2] with the following Butcher tableau: 
Table 1

Butcher Tableau for 7-stage fourth order RK method

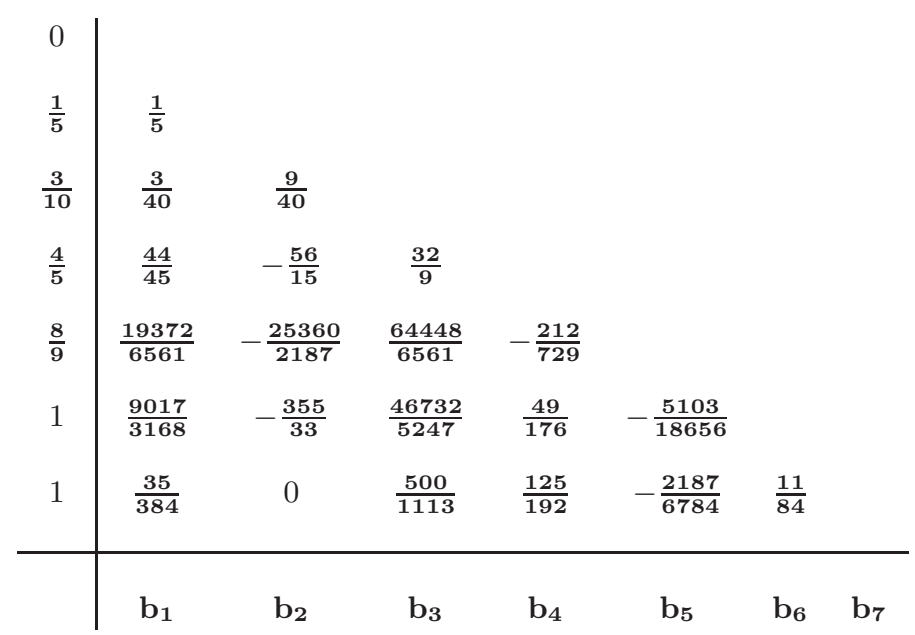

For this method, the phase-fitted and amplification-fitted conditions (13) become

$$
\begin{gathered}
1-z^{2}\left(\frac{1}{5} b_{2}(z)+\frac{3}{10} b_{3}(z)+\frac{4}{5} b_{4}(z)+\frac{8}{9} b_{5}(z)+b_{6}(z)+b_{7}(z)\right) \\
+z^{4}\left(\frac{4}{25} b_{4}(z)+\frac{1}{6} b_{7}(z)+\frac{424}{1215} b_{5}(z)+\frac{21}{55} b_{6}(z)\right) \\
-z^{6}\left(\frac{7}{550} b_{6}(z)+\frac{1}{120} b_{7}(z)\right)=\cos (z) \\
z\left(b_{1}(z)+b_{2}(z)+b_{3}(z)+b_{4}(z)+b_{5}(z)+b_{6}(z)+b_{7}(z)\right) \\
-z^{3}\left(\frac{9}{200} b_{3}(z)+\frac{8}{25} b_{4}(z)+\frac{32}{81} b_{5}(z)+\frac{1}{2} b_{6}(z)+\frac{1}{2} b_{7}(z)\right) \\
+z^{5}\left(\frac{-848}{18225} b_{5}(z)-\frac{14}{275} b_{6}(z)+\frac{1}{24} b_{7}(z)\right) \\
-z^{7}\left(\frac{1}{600} b_{6}(z)\right)=\sin (z)
\end{gathered}
$$


From equations (14), (15) and following sufficient conditions in [8], we obtain:

$$
\begin{gathered}
b^{T} e=b_{1}(z)+b_{2}(z)+b_{3}(z)+b_{4}(z)+b_{5}(z)+b_{6}(z)+b_{7}(z)=1, \\
b^{T} c=\frac{1}{5} b_{2}(z)+\frac{3}{10} b_{3}(z)+\frac{4}{5} b_{4}(z)+\frac{8}{9} b_{5}(z)+b_{6}(z)+b_{7}(z)=\frac{1}{2}, \\
b^{T} c^{2}=\frac{1}{25} b_{2}(z)+\frac{9}{100} b_{3}(z)+\frac{16}{25} b_{4}(z)+\frac{64}{81} b_{5}(z)+b_{6}(z)+b_{7}(z)=\frac{1}{3}, \\
b^{T} A c=\frac{9}{200} b_{3}(z)+\frac{8}{25} b_{4}(z)+\frac{32}{81} b_{5}(z)+\frac{1}{2} b_{6}(z)+\frac{1}{2} b_{7}(z)=\frac{1}{6}, \\
b^{T} c^{3}=\frac{1}{125} b_{2}(z)+\frac{27}{1000} b_{3}(z)+\frac{64}{125} b_{4}(z)+\frac{512}{729} b_{5}(z)+b_{6}(z)+b_{7}(z)=\frac{1}{4} .
\end{gathered}
$$

Solving (14), (15) and (16), we obtain:

$$
\begin{aligned}
& b_{1}(z)=\frac{5}{288} \frac{3665 z^{5}+346 z^{7}+606000 z-16970 z^{3}}{z^{5}\left(460+73 z^{2}\right)} \\
& b_{2}(z)=0, \\
& b_{3}(z)=-\frac{50}{3339} \frac{-8800 z^{5}-170400 \sin (z)-67880 z^{3}}{z^{5}\left(460+73 z^{2}\right)}, \\
& b_{4}(z)=\frac{25}{48} \frac{1825 z^{5}+54 z^{7}+606000 z-1697 z^{3}}{z^{5}\left(460+73 z^{2}\right)}, \\
& b_{5}(z)=-\frac{243}{1696} \frac{7285 z^{5}-22 z^{7}+303000 z-8485 z^{3}}{z^{5}\left(460+73 z^{2}\right)}, \\
& b_{6}(z)=-\frac{55}{42} \frac{-25680 z+614 z^{3}+426 z-8485 z^{5}}{z^{5}\left(460+73 z^{2}\right)}, \\
& b_{7}(z)=\frac{2\left(-9600 z+2000 z^{3}-100 z^{5}+7500 \sin (z)\right.}{z^{5}\left(460+73 z^{2}\right)} .
\end{aligned}
$$

As $z \rightarrow 0$, we obtain the following Taylor expansions:

$$
\begin{aligned}
b_{1}(z) & =\frac{35}{384}+\frac{145}{1112832} z^{2}-\frac{12761}{263264256} z^{4}+\frac{37347313}{4662409973760} z^{6}-\ldots \\
b_{2}(z) & =0 \\
b_{3}(z) & =\frac{500}{1113}-\frac{725}{1612737} z^{2}+\frac{9115}{54503928} z^{4}-\frac{37347313}{1351370390832} z^{6}+\ldots \\
b_{4}(z) & =\frac{125}{192}-\frac{725}{185472} z^{2}-\frac{63805}{43877376} z^{4}+\frac{37347313}{155413665792} z^{6}+\ldots \\
b_{5}(z) & =\frac{-2187}{6784}-\frac{11745}{2184448} z^{2}+\frac{114849}{57419776} z^{4}-\frac{336125817}{1016904232960} z^{6}+\ldots
\end{aligned}
$$




$$
\begin{gathered}
b_{6}(z)=\frac{11}{84}+\frac{2321}{405720} z^{2}-\frac{646217}{479908800} z^{4}+\frac{168275831}{772653168000} z^{6}-\ldots, \\
b_{7}(z)=\frac{-19}{4830} z^{2}+\frac{13633}{19996200} z^{4}-\frac{3640283}{33726924000} z^{6}+\ldots .
\end{gathered}
$$

The result is compared, then we analysed which problem will give the small error. This new method is denoted as PHAFRK4D.

Using equation (6), we obtained the stability polynomial are three different stages of the solutions. First, we take the value of $b_{1}, b_{2}, b_{3}, b_{4}, b_{5}, b_{6}$ and $b_{7}$ up to $h^{6}$ from their series solution.

$$
\begin{aligned}
b_{1}(z)= & \frac{35}{384}+\frac{145}{1112832} z^{2}-\frac{12761}{263264256} z^{4}+\frac{37347313}{4662409973760} z^{6} \\
b_{2}(z)= & 0 \\
b_{3}(z)= & \frac{500}{1113}-\frac{725}{1612737} z^{2}+\frac{9115}{54503928} z^{4}-\frac{37347313}{1351370390832} z^{6} \\
b_{4}(z)= & \frac{125}{192}-\frac{725}{185472} z^{2}-\frac{63805}{43877376} z^{4}+\frac{37347313}{155413665792} z^{6} \\
b_{5}(z)= & \frac{-2187}{6784}-\frac{11745}{2184448} z^{2}+\frac{114849}{57419776} z^{4}-\frac{3336125817}{1016904232960} z^{6} \\
b_{6}(z)= & \frac{11}{84}+\frac{2321}{405720} z^{2}-\frac{646217}{479908800} z^{4}+\frac{168275831}{772653168000} z^{6} \\
b_{7}(z)= & \frac{-19}{4830} z^{2}+\frac{13633}{19996200} z^{4}-\frac{3640283}{33726924000} z^{6} \\
R(z)=1+z+ & \frac{1}{2} \hat{z}^{2}+\frac{1}{6} \hat{z}^{3}+\frac{1}{24} \hat{z}^{4}+\frac{1}{120} \hat{z}^{5}+\frac{7}{3600} \hat{z}^{6}-\frac{33}{161000} \hat{z}^{7}+\frac{2431}{23184000} \hat{z}^{8} \\
- & \frac{63929}{23995440000} \hat{z}^{9}-\frac{1112821}{47990880000} \hat{z}^{10}+\frac{7067657}{758855790000} \hat{z}^{11} \\
& +\frac{8119399}{4336318800000} \hat{z}^{12}-\frac{3640283}{20236154400000} \hat{z}^{13}+\ldots
\end{aligned}
$$

Secondly, we take the values $b_{1}, b_{2}, b_{3}, b_{4}, b_{5}, b_{6}$ and $b_{7}$ up to $h^{8}$ from their series solution.

$$
\begin{gathered}
b_{1}(z)=\frac{35}{384}+\frac{145}{1112832} z^{2}-\frac{12761}{263264256} z^{4}+\frac{37347313}{4662409973760} z^{6} \\
-\frac{106536829171}{83643634929254400} z^{8}
\end{gathered}
$$

$$
b_{2}(z)=0
$$




$$
\begin{gathered}
b_{3}(z)=\frac{500}{1113}-\frac{725}{1612737} z^{2}+\frac{9115}{54503928} z^{4}-\frac{37347313}{1351370390832} z^{6} \\
+\frac{106536829171}{24243584811526080} z^{8}
\end{gathered}
$$

$$
\begin{gathered}
b_{4}(z)=\frac{125}{192}-\frac{725}{185472} z^{2}-\frac{63805}{43877376} z^{4}+\frac{37347313}{155413665792} z^{6} \\
+\frac{106536829171}{2788121164308480} z^{8}
\end{gathered}
$$

$$
\begin{gathered}
b_{5}(z)=\frac{-2187}{6784}-\frac{11745}{2184448} z^{2}+\frac{114849}{57419776} z^{4}-\frac{336125817}{1016904232960} z^{6} \\
+\frac{319610487513}{608108731300800} z^{8}
\end{gathered}
$$

$$
\begin{gathered}
b_{6}(z)=\frac{11}{84}+\frac{2321}{405720} z^{2}-\frac{646217}{479908800} z^{4}+\frac{168275831}{772653168000} z^{6} \\
-\frac{479440217357}{13861397833920000} z^{8}
\end{gathered}
$$

$$
\begin{gathered}
b_{7}(z)=\frac{-19}{4830} z^{2}+\frac{13633}{19996200} z^{4}-\frac{3640283}{33726924000} z^{6}+\frac{31076448353}{1815183049680000} z^{8} \\
R(z)=1+z+\frac{1}{2} \hat{z}^{2}+\frac{1}{6} \hat{z}^{3}+\frac{1}{24} \hat{z}^{4}+\frac{1}{120} \hat{z}^{5}+\frac{7}{3600} \hat{z}^{6}-\frac{33}{161000} \hat{z}^{7}+\frac{2431}{23184000} \hat{z}^{8} \\
-\frac{63929}{23995440000} \hat{z}^{9}-\frac{1112821}{47990880000} \hat{z}^{10}+\frac{7067657}{758855790000} \hat{z}^{11} \\
\quad+\frac{2729638939}{728501558400000} \hat{z}^{12}-\frac{27444780347}{181518304968000000} \hat{z}^{13} \\
-\frac{3115942073}{10472209902000000} \hat{z}^{14}+\frac{31076448353}{1089109829808000000} \hat{z}^{15}+\ldots
\end{gathered}
$$


Lastly, we take the values $b_{1}, b_{2}, b_{3}, b_{4}, b_{5}, b_{6}$ and $b_{7}$ up to $h^{10}$ from their series solution,

$$
\begin{aligned}
& b_{1}(z)=\frac{35}{384}+\frac{145}{1112832} z^{2}-\frac{12761}{263264256} z^{4}+\frac{37347313}{4662409973760} z^{6} \\
& -\frac{106536829171}{83643634929254400} z^{8}-\frac{81666254778209}{403998756708298752000} z^{10} \\
& b_{2}(z)=0 \\
& b_{3}(z)=\frac{500}{1113}-\frac{725}{1612737} z^{2}+\frac{9115}{54503928} z^{4}-\frac{37347313}{1351370390832} z^{6} \\
& +\frac{106536829171}{24243584811526080} z^{8}-\frac{81666254778209}{117096514639670966400} z^{10} \\
& b_{4}(z)=\frac{125}{192}-\frac{725}{185472} z^{2}-\frac{63805}{43877376} z^{4}+\frac{37347313}{155413665792} z^{6} \\
& +\frac{106536829171}{2788121164308480} z^{8}-\frac{81666254778209}{13466625223609958400} z^{10} \\
& b_{5}(z)=\frac{-2187}{6784}-\frac{11745}{2184448} z^{2}+\frac{114849}{57419776} z^{4}-\frac{336125817}{1016904232960} z^{6} \\
& +\frac{319610487513}{608108731300800} z^{8}-\frac{81666254778209}{9790550574092288000} z^{10} \\
& b_{6}(z)=\frac{11}{84}+\frac{2321}{405720} z^{2}-\frac{646217}{479908800} z^{4}+\frac{168275831}{772653168000} z^{6} \\
& -\frac{479440217357}{13861397833920000} z^{8}-\frac{367498709681953}{66950551537833600000} z^{10} \\
& b_{7}(z)=\frac{-19}{4830} z^{2}+\frac{13633}{19996200} z^{4}-\frac{3640283}{33726924000} z^{6}+\frac{31076448353}{1815183049680000} z^{8} \\
& -\frac{3664522778473}{1348820635377600000} z^{10}
\end{aligned}
$$




$$
\begin{gathered}
R(z)=1+z+\frac{1}{2} \hat{z}^{2}+\frac{1}{6} \hat{z}^{3}+\frac{1}{24} \hat{z}^{4}+\frac{1}{120} \hat{z}^{5}+\frac{7}{3600} \hat{z}^{6}-\frac{33}{161000} \hat{z}^{7}+\frac{2431}{23184000} \hat{z}^{8} \\
-\frac{63929}{23995440000} \hat{z}^{9}-\frac{1112821}{47990880000} \hat{z}^{10}+\frac{7067657}{758855790000} \hat{z}^{11}+\frac{2729638939}{728501558400000} \hat{z}^{12} \\
-\frac{27444780347}{181518304968000000} \hat{z}^{13}-\frac{9073798217201}{15247537617312000000} \hat{z}^{14} \\
+\frac{22959240867371}{956436450540480000000} \hat{z}^{15}+\frac{165601146254387}{3506933651981760000000} \\
-\frac{3664522778473}{809292381226560000000} \hat{z}^{16}+\ldots
\end{gathered}
$$

we next obtained the stability region of the new method from the above three stability polynomials by equating each to the Euler formula and then solve for $h$ using maple package.

i.e

$$
R(\hat{h})=e^{I \theta}=\cos (\theta)+I \sin (\theta) .
$$

The stability region for the new method is shown in Figure 1.

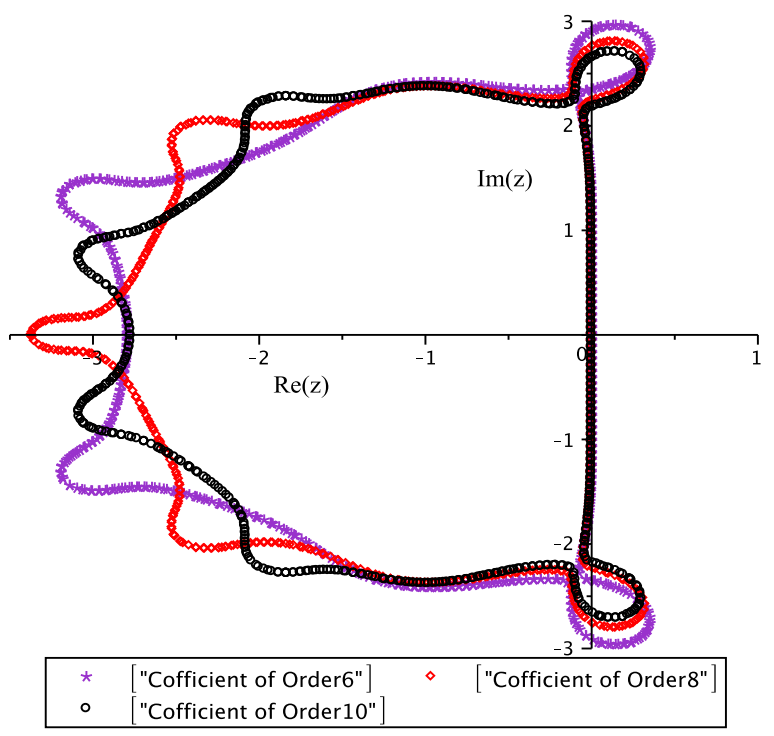

Figure 1: The stability region for the new method PHAFRK4D 
Definition. A Runge-kutta method is said to be absolutely stable if $\forall \hat{h} \epsilon(-h, 0)$, $|R(\hat{h})|<1$.

Now, our new method is absolutely stable since for all $\hat{h} \epsilon(-6.9,0)$, $|R(\hat{h})|<1$ where we obtained using maple package.

\subsection{Error Analysis}

In this section, we will compute the local truncation error analysis (LTE) of the new method is based on the Taylor series expansion of the differences $y_{n+1}$ and $y\left(x_{n}+h\right)$

$$
\begin{gathered}
L T E=y_{n+1}-y\left(x_{n}+h\right) \\
L T E=h^{5}\left[-\frac{71}{6480000}\left(f_{x x x x}+f^{4} f_{y y y y}\right)-\frac{191}{1080000} y^{(2)} f_{y} f_{x y y}\right. \\
-\frac{1}{54000}\left(131 y^{\prime} f_{y} f_{x x y}-71 y^{(2)} f_{x y} f_{y y}-71 y^{\prime} f_{x} f_{x y y}\right)+\frac{11}{4000} f_{y}^{2} f_{x y} y^{\prime} \\
-\frac{1}{27000} f_{y} f_{y y y} y^{(3)}-\frac{1}{360000}\left(173 f_{x} f_{y} f_{x y}-37 f_{y} f_{x x x}-37 f_{y}^{2} f_{x x}\right) \\
+\frac{71}{1080000}\left(f_{x} f_{y y y} y^{(2)}+f_{x x y y} y^{(2)}+f_{x} f_{x x y}\right) \\
\frac{71}{1620000}\left(f_{x x x y}^{\prime}+f_{x x y y} y^{(2)}+f_{x y y y} y^{(3)}+f_{x x} f_{x y}+f_{y y}^{2} f^{(3)}\right)+\frac{71}{810000} f_{x y}^{2} y^{\prime} \\
\frac{1}{2160000}\left(887 f_{y}^{2} f_{y y} y^{(2)}+71 f_{x}^{2} f_{y y}\right) \\
\left.\left.-\frac{97}{120000}\left(f_{y}^{3} f_{x}+f^{4} y^{\prime}\right)+\frac{59}{108000} f_{x} f_{y} f_{y y} y^{\prime}\right)\right]+O\left(h^{6}\right) .
\end{gathered}
$$

From equation (23), it is clear that the order of the new method is four because all the terms of $h$ lower than $h^{5}$ are vanished.

\section{Numerical Results}

In this section, we will apply the new method to solve differential equations (1). The following explicit RK methods are selected for the numerical comparison.

- PHAFRK4D: the seven-stage fourth-order phase-fitted and amplificationfitted RK method given in Section 3 of this paper. 
- PLRK4: The modified RK method derived by Simos and Vigo-Aguiar [9].

- RK5B: Fifth-order six-stage RK method derived by Sakak and Simos [10].

- RK4M: Fourth-order five-stage RK method given in Butcher [11].

- PHRK5: six-stage fifth-order modified RK method given by Simos and Vigo-Aguiar [12].

Problem 1: ( Homogeneous)

$$
\begin{gathered}
y_{1}^{\prime}=y_{2}, y_{1}(x)=1 \\
y_{2}^{\prime}=-64 y_{1}, y_{2}(x)=-2
\end{gathered}
$$

Theoretical solution :

$$
\begin{aligned}
& y_{1}(x)=-\frac{1}{4} \sin (8 x)+\cos (8 x) \\
& y_{2}(x)=-2 \cos (8 x)-8 \sin (8 x)
\end{aligned}
$$

Source : Chawla and Rao [13].

Problem 2: (Inhomogeneous)

$$
\begin{gathered}
y_{1}^{\prime}=y_{2}, y_{1}(x)=1 \\
y_{2}^{\prime}=-v^{2} y_{1}+\left(v^{2}-1\right) \sin (x), y_{2}(x)=v+1
\end{gathered}
$$

Estimated frequency: $v=10$.

Theoretical solution :

$$
\begin{gathered}
y(x)=\cos (v x)+\sin (v x)+\sin (x) \\
y_{2}(x)=-v \sin (v x)+v \cos (v x)+\cos (x)
\end{gathered}
$$

Source :Van der Houwen and Sommeijer [6].

Problem 3: (Periodic orbit system)

$$
\begin{gathered}
y_{1}^{\prime}=y_{3}, y_{1}(x)=1 \\
y_{3}^{\prime}=-y_{1}+0.001 \cos (x), y_{3}(x)=0 \\
y_{2}^{\prime}=y_{4}, y_{2}(x)=0
\end{gathered}
$$




$$
y_{4}^{\prime}=-y_{2}+0.001 \sin (x), y_{4}(x)=0.9995
$$

Theoretical solution:

$$
\begin{gathered}
y_{1}(x)=\cos (x)+0.0005 x \sin (x) \\
y_{2}(x)=\sin (x)-0.0005 x \cos (x) \\
y_{3}(x)=-\sin (x)+0.0005 x \cos (x) \\
y_{4}(x)=\cos (x)+0.0005 x \sin (x)
\end{gathered}
$$

Source : Stiefel and Bettis [14].

Problem 4: (Nonlinear system)

$$
\begin{gathered}
y_{1}^{\prime}=y_{3}, y_{1}(0)=1 \\
y_{3}^{\prime}=\frac{-y_{1}}{\left(\sqrt{y_{1}^{2}+y_{2}^{2}}\right)^{3}}, y_{3}(0)=0 \\
y_{2}^{\prime}=y_{4}, y_{2}(0)=0 \\
y_{4}^{\prime}=\frac{-y_{2}}{\left(\sqrt{y_{1}^{2}+y_{2}^{2}}\right)^{3}}, y_{4}(0)=1
\end{gathered}
$$

Theoretical solution :

$$
\begin{gathered}
y_{1}(x)=\cos (x) \\
y_{2}(x)=\sin (x) \\
y_{3}(x)=-\sin (x) \\
y_{4}(x)=\cos (x)
\end{gathered}
$$

Source : Moo et. al [15].

Problem 5: (Inhomogeneous system)

$$
\begin{gathered}
y_{1}^{\prime}=y_{3}, y_{1}(x)=1.1 \\
y_{3}^{\prime}=-16 y_{1}+16 e^{-10 x}+100 e^{-10 x}, y_{3}(x)=-10 \\
y_{2}^{\prime}=y_{4}, y_{2}(x)=1
\end{gathered}
$$




$$
y_{4}^{\prime}=-16 y_{2}+16 e^{-10 x}+100 e^{-10 x}, y_{4}(x)=-9.6
$$

Theoretical solution :

$$
\begin{aligned}
& y_{1}=0.1 \cos (4 x)+e^{-10 x} \\
& y_{2}=0.1 \sin (4 x)+e^{-10 x} \\
& y_{3}=-0.4 \sin (4 x)-10 e^{-10 x} \\
& y_{4}=0.4 \cos (4 x)-10 e^{-10 x}
\end{aligned}
$$

Source : Moo. et. al [15].

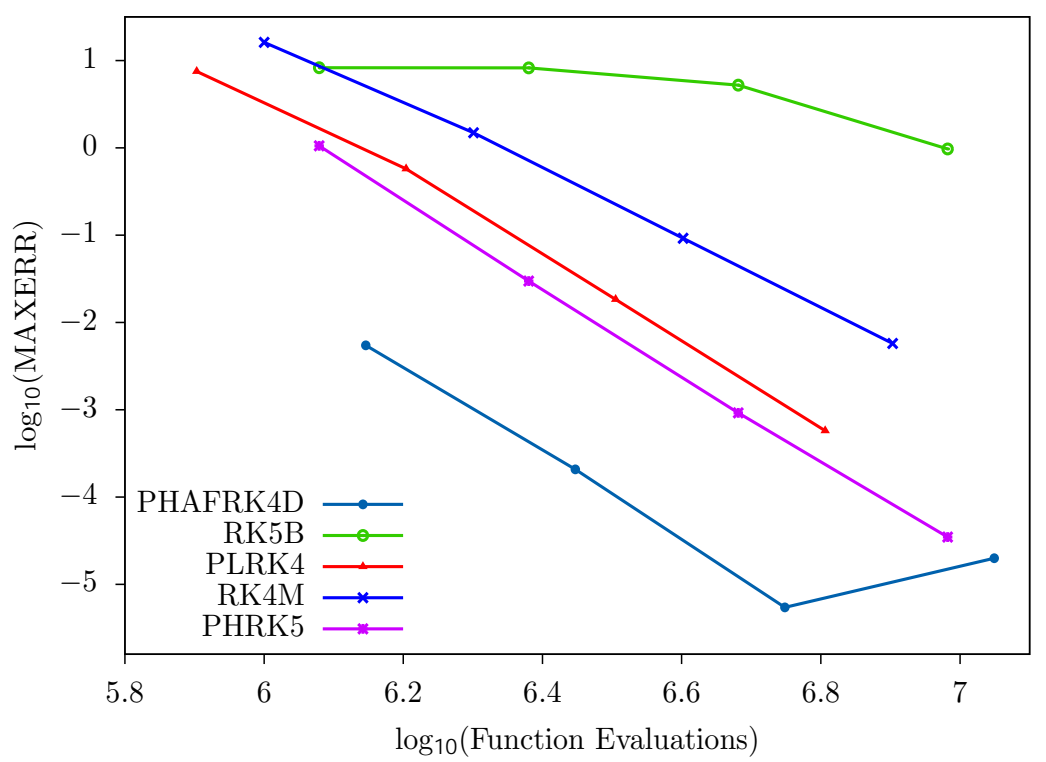

Figure 2: Efficiency curves of all the methods for problem 1 with $h=0.00625,0.0125,0.025$ and 0.05 for $b=10000$

\section{Discussion and Conclusion}

In this study, we have presented a new phase-fitted and amplification-fitted (PHAFRK4D) method that can be used to solve first-order ordinary differential equations with the solutions are oscillating. The numerical results are plotted in Figures 2, 3, 4, 5 and 6. Those Figures display the efficiency curves where the common logarithm of the maximum global absolute error throughout the integration versus computational cost measured by number of function 


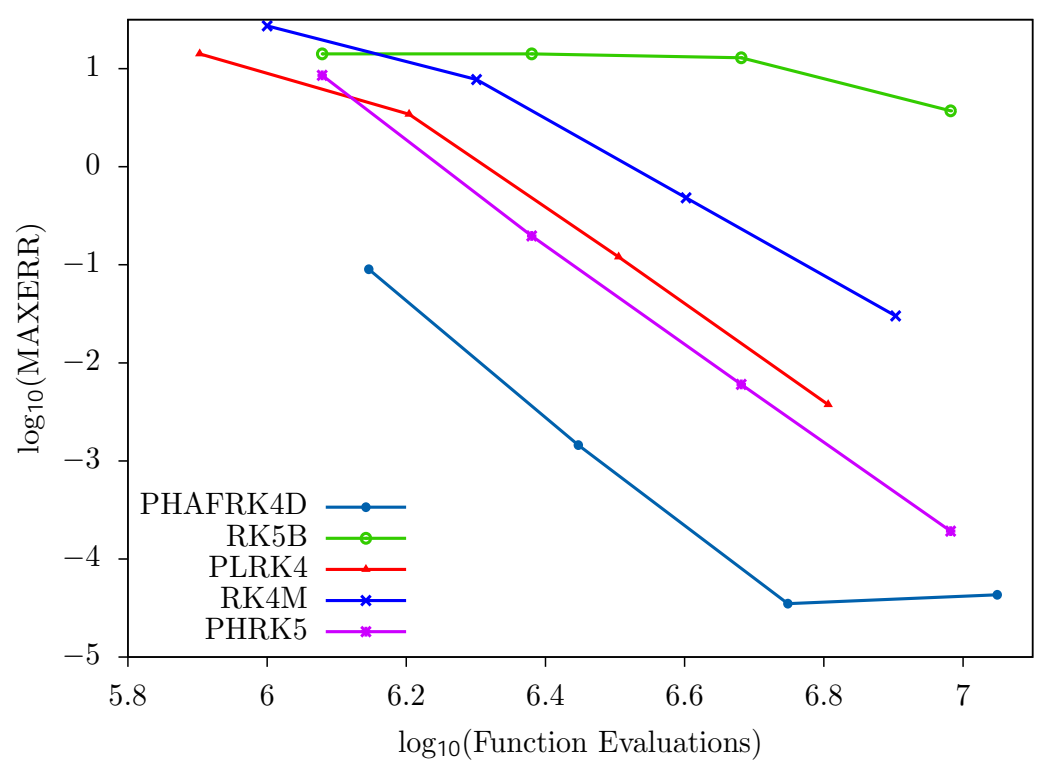

Figure 3: Efficiency curves of all the methods for problem 2 with $h=0.00625,0.0125,0.025$ and 0.05 for $b=10000$

evaluations. This new method is based on fourth-order seven-stage explicit Runge-Kutta. From Figures 2 to 6, the numerical results we got it indicate the robustness of the new method in comparison with other existing methods. 


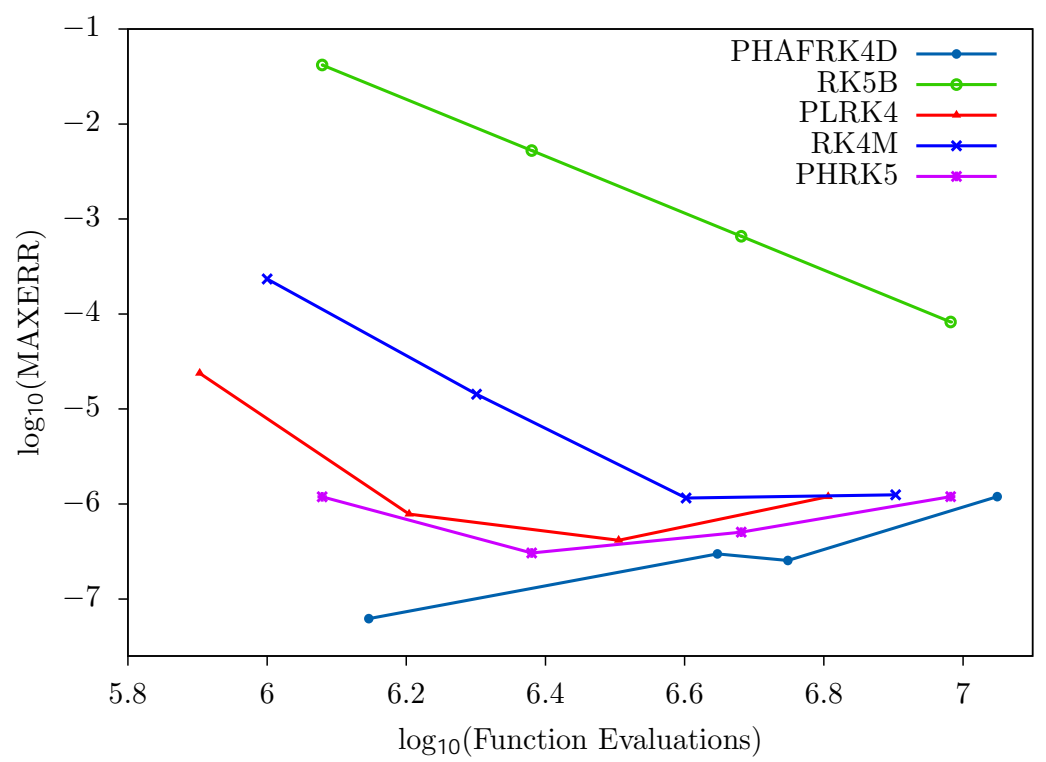

Figure 4: Efficiency curves of all the methods for problem 3 with $h=0.00625,0.0125,0.025$ and 0.05 for $b=10000$

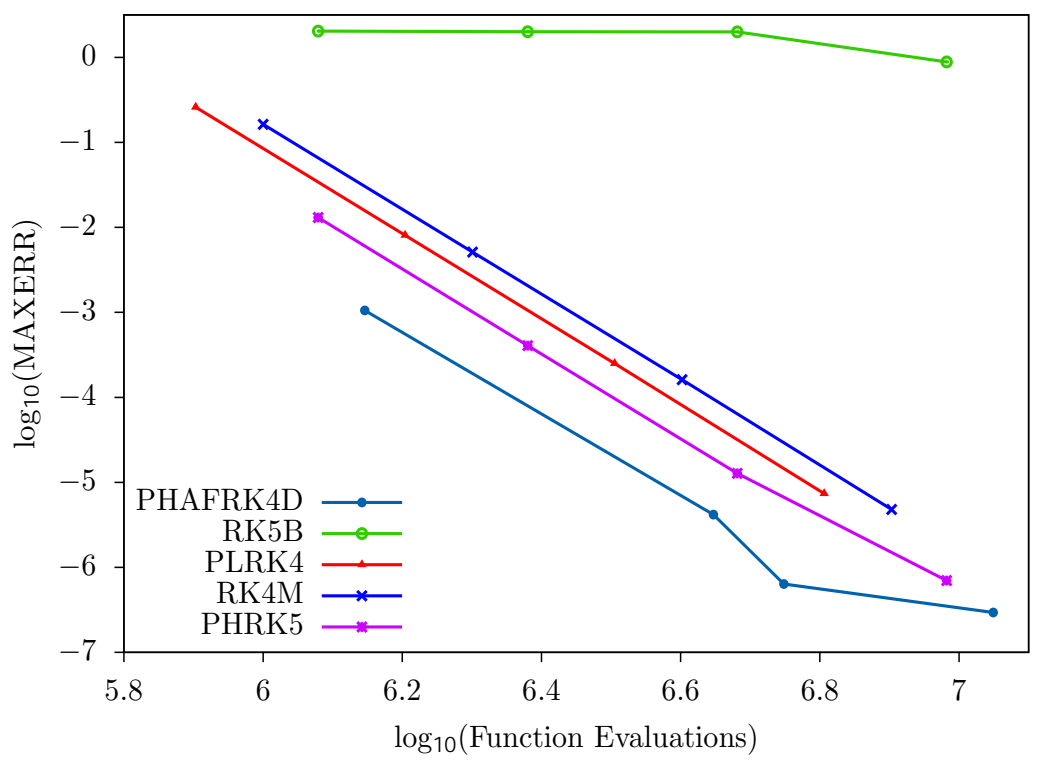

Figure 5: Efficiency curves of all the methods for problem 4 with $h=0.00625,0.0125,0.025$ and 0.05 for $b=10000$ 


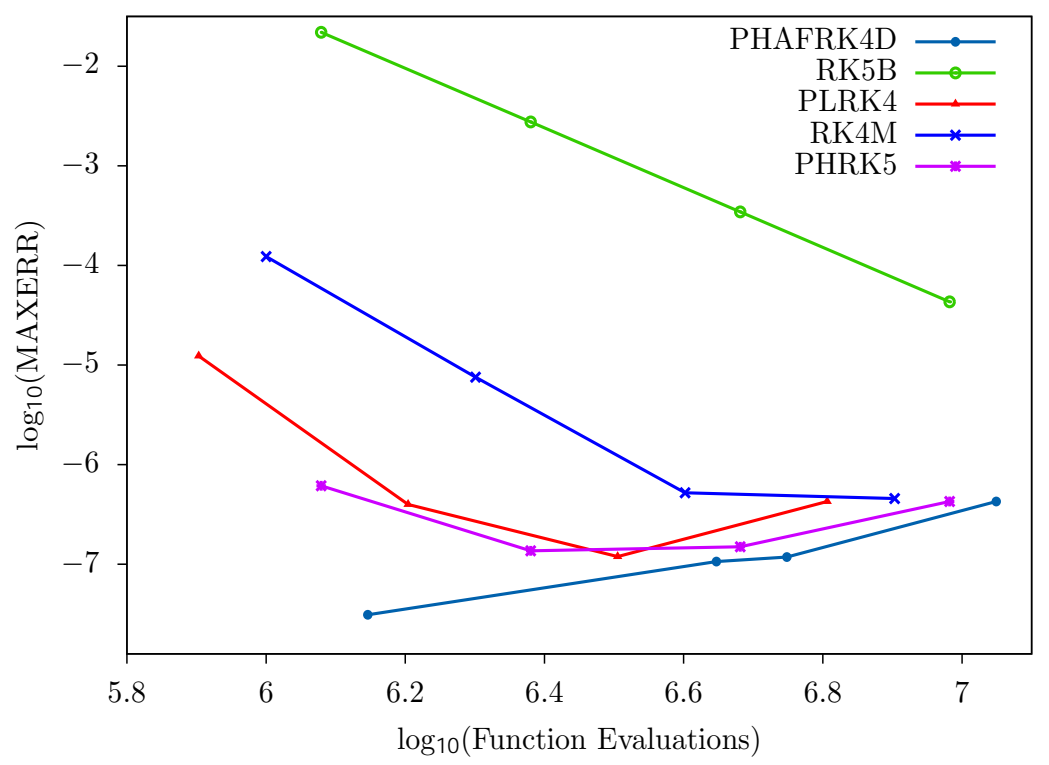

Figure 6: Efficiency curves of all the methods for problem 5 with $h=0.00625,0.0125,0.025$ and 0.05 for $b=10000$ 


\section{References}

[1] D. G. Bettis, Runge-Kutta Algorithms for Oscillatory Problems, Journal of Applied Mathematics and Physics, 30, No. 4 (1979), 699-704.

[2] J. M. Franco, Runge-Kutta-Nyström methods adapted to the numerical integration of perturbed oscillators, Computer Physics Communications, 147, No. 3 (2002), 770-787.

[3] Z. A. Anastassi and T. E. Simos, A dispersive-fitted and dissipative-fitted explicit RungeKutta method for the numerical solution of orbital problems, New Astronomy, 10, No. 1 (2004), 31-37.

[4] E. Hairer, S. P. Nørsett and G. Wanner, Solving Ordinary Differential Equations I, Non stiff Problems, Springer, Germany (1993).

[5] J. Vigo-Aguiar and T. E. Simos, An exponentially-fitted and trigonometrically-fitted method for the numerical solution of orbital problems, Astronomical Journal, 122, No. 3 (2001), 1656-1660.

[6] P. J. Van der Houwen, B. P. Sommeijer, Explicit Runge-Kutta-Nyström methods with reduced phase errors for computing oscillating solutions, SIAM J. Numer. Anal., 24, No. 3 (1987), 595-617.

[7] B. Paternoster, Runge-Kutta (Nystrom) methods for ODEs with periodic solutions based on trigonometric polynomials, Applied Numerical Mathematics, 28, No. 24 (1998), 401412.

[8] J. M. Franco, Runge-Kutta methods adapted to the numerical integration of oscillatory problem, Applied Numerical Mathmatics, 50, No. 3-4 (2004), 427-443.

[9] T. E. Simos, J. V. Aguiar, A modified Runge-Kutta method with phase-lag of order infinity for the numerical solution of the Schrödinger equation and related problems, Computers $\& 3$ chemistry, 25, No. 3 (2001), 275-281.

[10] D. P. Sakas, T.E. Simos, A Fifth Order Trigonometrically-Fitted Modified Runge-Kutta Zonneveld Method for the Numerical Solution of Orbital problems, Mathematical and Computer Modelling, 42, No. 7-8 (2005), 903-920.

[11] J. C. Butcher, Numerical Methods for Ordinary Differential Equations, John Wiley \& Sons Ltd, England (2008).

[12] T. E. Simos and J. V. Aguiar, A modified phase-fitted Runge-Kutta method for the numerical solution of the Schrödinger equation, J. Math. Chem., 30, No. 1 (2001), 121131.

[13] M. M. Chawla, P. S. Rao, High-accuracy P-stable methods $y^{\prime \prime}=f(t, y)$, IMA J Numer Anal, 5, No. 2 (1985), 215-220.

[14] E. Stiefel, D. G. Bettis, An invariant imbedding algorithm for the solution of inhomogeneous linear two-point boundary value problems, Journal of Computational Physics, 14, No. 1 (1974), 40-48.

[15] K. W. Moo, N. Senu, F. Ismail and M. Suleiman, New phase-fitted and amplificationfitted fourth-order and fifth-order Runge-Kutta-Nyström methods for oscillatory problems, Hindawi Publishing Corporation, 2013, Article ID 939367 (2013), 9 pages. 